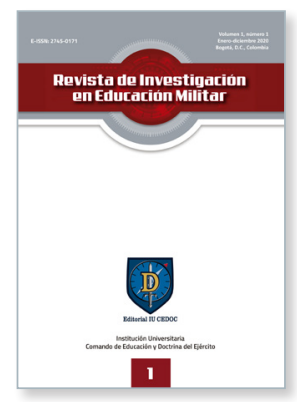

Revista de Investigación en Educación Militar

Volumen 1, número 1, enero-diciembre 2020, pp. 27-50

Bogotá, D. C., Colombia

E-ISSN: 2745-0171

https://doi.org/10.47961/27450171.4

\title{
Didáctica de la historia en la educación superior a través de las mediaciones. De la Historia Estelar a la Educación Estelar
}

\section{Robert Manuel Ojeda Pérez}

https://orcid.org/0000-0002-1227-7854

rojeda@unisalle.edu.co

Universidad de La Salle, Bogotá D. C., Colombia

Citación: Ojeda Pérez, R. M. (2020). Didáctica de la historia en la educación superior a través de las mediaciones. De la Historia Estelar a la Educación Estelar. Revista de Investigación en Educación Militar, 1(1), 27-50. DOI: https://doi. org/10.47961/27450171.4

Publicado en línea: $1 .^{\circ}$ de diciembre de 2020

Los artículos publicados por la Revista de Investigación en Educación Militar son de acceso abierto bajo una licencia Creative Commons: Atribución - No Comercial - Sin Derivados.

Para enviar un artículo:

https://revistascedoc.com/index.php/riem/about/submissions

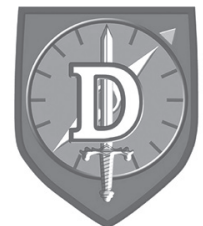

Editorial IU CEDOC 


\title{
Didáctica de la historia en la educación superior a través de las mediaciones. De la Historia Estelar a la Educación Estelar
}

\author{
Didactics of the history in higher education through \\ mediations. From Stellar History to Stellar Education
}

\author{
Robert Manuel Ojeda Pérez \\ Universidad de La Salle, Bogotá D. C., Colombia
}

\section{Resumen}

La enseñanza de la historia en Colombia evidencia los distintos enfoques con los que se han divulgado las investigaciones en esta área. Sin embargo, son pocos los trabajos que se han hecho en el país sobre la didáctica de la investigación y la enseñanza de este campo. Este artículo hace una revisión documental sobre la enseñanza de la historia en Colombia y presenta un estudio de caso en el que se propone una didáctica desde la Historia Estelar en un espacio académico propio denominado "Narraciones para el posconflicto". Específicamente, se orienta a los estudiantes de primer semestre de Negocios y Relaciones Internacionales de la Universidad de La Salle en el proceso de hacer una investigación a manera de monografía para presentar un artículo en una revista de divulgación académica universitaria indexada. Los resultados evidencian un cambio en las narrativas, en los procesos de enseñanza y aprendizaje, y la escritura de artículos de investigación históricos de la comunidad académica implicada.

Palabras clave: didáctica de la historia; educación superior; enseñanza de la historia; Historia Estelar; mediaciones.

\section{Abstract}

The teaching of history in Colombia shows the different approaches with which research in this area has been disseminated. However, few studies have been done in the country on the teaching of research and teaching in this field. This article makes a documentary review on the teaching of history in Colombia and presents a case study in which a didactics from Stellar History is proposed in its own academic space called "Narrations for the post-conflict". Specifically, it is aimed to the first semester students of Business and International Relations of the University of La Salle in the process of doing a research as a monograph to present an article in an indexed university academic journal. The results show a change in the narratives, in the teaching and learning processes, and the writing of historical research articles of the academic community involved.

Keywords: didactics of history; higher education; history teaching; mediations; Stellar History.

Artículo de reflexión

Recibido: 1 de mayo de 2020

Aceptado: 20 de agosto de 2020

Contacto: Robert Manuel Ojeda Pérez $\square$ rojeda@unisalle.edu.co 


\section{Antecedentes y planteamiento del problema}

Esta propuesta se centra en la didáctica de la historia en la educación superior. Específicamente, parte del análisis y la reflexión de un estudio de caso para formar en el reconocimiento del otro las didácticas y las pedagogías en la enseñanza de la historia a partir de las mediaciones y la acción participativa.

Gran parte de la problemática de la enseñanza de la historia en el nivel superior es su conceptualización. Al respecto, la Comisión de Historia del Proyecto Alfa Tuning para América Latina señala lo siguiente:

La enseñanza universitaria de la Historia tiene una larga tradición en América Latina. Ha sido, y sigue siendo, parte sustantiva del currículo de carreras diversas, como Derecho, Sociología, Antropología, Relaciones Internacionales, entre otras. Paralelamente, experimentó, a lo largo del siglo XX, un desarrollo importante como carrera autónoma en el seno de facultades de Humanidades o Filosofía y Letras. En la mayoría de los casos, este nacimiento fue el producto de un proceso de diferenciación paulatina de programas que incluían la Historia como materia central, o como orientación de las tesis de graduación. Constituidos como desprendimientos de dichas carreras originales, los departamentos y carreras de Historia nacieron siguiendo una cronología que se extiende desde fines del siglo XIX hasta periodos muy recientes. (Tuning, 2007)

El mismo proyecto Alfa Tuning destaca que la historia es la única disciplina capaz de proporcionar un conocimiento racional y crítico del pasado, que permite comprender el presente; que pone en relación los acontecimientos del pasado con la actualidad; que contribuye al reconocimiento, la tolerancia y el respeto de la diversidad cultural; fomenta el desarrollo de una conciencia cívica y ayuda a identificar los procesos de cambio y de continuidad en una perspectiva diacrónica.

Sin embargo, esta necesidad de visibilizar la historia como disciplina autónoma es relativamente tardía en América Latina. A pesar de que en este proyecto no se quiere hacer una historia de la educación en Colombia, ni tampoco una historia de su enseñanza, es bueno tener en cuenta el contexto colombiano para saber cómo se ha desarrollado la historiografía y de esta manera poder proponer un modelo de investigación y de enseñanza que posibilite el reconocimiento del otro dentro de la narrativa histórica.

El nuevo perfil de un estudiante de historia en educación superior fue desarrollado por la Comisión Tuning para América Latina 2013, en la cual incluyeron a Colombia. Específicamente, señala que los egresados deben tener las siguientes competencias:

El egresado de la carrera de Historia es un especialista en el conocimiento y la comprensión crítica del pasado humano, que entiende la Historia como una ciencia en permanente construcción en diálogo con otras ciencias. Conoce las principales corrientes y teorías his- 
toriográficas; es capaz de emplear metodologías y técnicas para producir conocimiento, comunicarlo y transferirlo con autonomía y responsabilidad a diversas audiencias. Comprende y realiza sus funciones profesionales, contribuyendo a la formación de una sociedad basada en valores ciudadanos y respetuosa de la diversidad cultural. (Tuning, 2013, p. 37)

Para obtener este perfil es indispensable que los trabajos en educación superior le apunten a desarrollar competencias en los estudiantes que posibiliten el logro de esta meta. Por tal motivo, esta investigación enfatiza la formación de una sociedad basada en valores y respetuosa de la diversidad cultural, así como el desarrollo del reconocimiento del otro. Específicamente, pretende mostrar las otras formas de enseñar la historia a partir de la narración y el empleo de las mediaciones como una didáctica en la que pueden participar los mismos estudiantes.

\section{Didáctica de la historia}

La investigación histórica ha mostrado un avance importante, aunque, como señala Jorge Orlando Melo (1999), la abundancia de publicaciones "cubre un abanico temático cada vez más amplio, sobre todo en los historiadores más jóvenes" (p. 20). Respecto a los historiadores con trayectoria, sus trabajos reflejan madurez en la escogencia de sus objetos de estudio y en los enfoques teóricos utilizados: "Son una muestra de la vitalidad del trabajo histórico que se hace en el país" (Melo, 1999, p. 21). Aunque todavía esta sigue siendo insular, no se proyecta como debería hacia la enseñanza básica y menos hacia el público en general.

Y es que a pesar de los avances señalados, no podemos obviar el remezón que la historia y, en general, las ciencias sociales vivieron a finales de los ochenta y los noventa. Eventos como la caída del Muro de Berlín, que evidenciaron la crisis del socialismo, la globalización neoliberal y su impacto en lo socio-cultural, además de los propios cuestionamientos que ha traído a la disciplina su acercamiento a la antropología, la lingüística, la semiología y la crítica literaria, se han reflejado en la apertura hacia corrientes posmodernas. Esta apertura ha sido vista con preocupación por algunos, mientras que otros la consideran una oportunidad para el cuestionamiento y el debate teórico, dos aspectos necesarios en la vida interna de una disciplina y más aún en la historia, por la relación y compromiso que ha tenido y tiene con los destinos de la sociedad.

Lo cierto es que la disciplina histórica se ha consolidado en nuestro país. Así lo evidencia el hecho de que los pregrados, maestrías y doctorados son apreciados por estudiantes y profesionales para realizar estudios, y es que a pesar de las crisis y gracias a ellas, para los hombres y las mujeres la historia sigue y seguirá ocupando un lugar determinante en sus vidas. El gran interrogante que puede surgir después de este recorrido es: ¿Cómo se enseña la historia en la educación superior? ¿Cuál puede ser la didáctica de la historia para formar ciudadanos con una sensibilidad y reconocimiento del otro? ¿Cuáles han sido las narrativas utilizadas? Es 
por ello que se hace necesario inculcar en las nuevas generaciones una conciencia histórica capaz de aceptar las incertidumbres y asumir los riesgos, para enfrentar todo tipo de problemas, sobre todo la inclusión del otro, el reconocimiento de un nosotros y la posibilidad de que ellos mismos pueden reconstruir la historia y narrarla desde su mismo locus de enunciación, con sus nuevos lenguajes tecnológicos.

La necesidad de reflexionar cómo se enseña la historia en el nivel superior es imperativa, ya que Colombia presenta necesidades sentidas en el reconocimiento del otro en la reconstrucción de la realidad social. Precisamente sobre este tema se encuentra una tesis de maestría de la Universidad de Caldas, La enseñanza de la historia: perspectivas y retos en la educación superior. Estudio de caso: enseñanza de la historia en la Licenciatura en Ciencias Sociales de la Universidad de Caldas (Posada, 2010). Esta tesis y su artículo hacen un análisis de la historia de Colombia desde la revisión de diversos hechos fundamentales para el desarrollo social, político y económico del país.

En dicho análisis se describe la situación de incertidumbre que ha ubicado a Colombia como el segundo país en conflicto armado y como uno de los países más violentos del mundo. Estas perspectivas posibilitan la reflexión del ejercicio de la vida en convivencia y del mundo de lo cotidiano, que se vislumbra como una experiencia de grandes encrucijadas y disyuntivas enmarcadas por una génesis de caracteres violentos, el indebido ejercicio de la política, la corrupción, la ausencia de Estado y la falta de fundamentos axiológicos. Precisamente por estas condiciones, la educación debería formar a los colombianos para admitir el disenso, acercar al consenso y reafirmar valores como la tolerancia, la diferencia, la reconciliación y el perdón en el proceso de formación de los estudiantes de historia y profesionales de la educación superior (Ojeda, 2018).

Para el caso mexicano, Andrea Sánchez Quintanar, historiadora y profesora de historia, sostiene en su trabajo que "los problemas de la enseñanza de la historia deben ser propuestos y resueltos por los historiadores" (Quintanar, 1995). Esta afirmación, a pesar de que invalida la ayuda y el diálogo con otras disciplinas, reconoce que la historia necesita reflexionarse como disciplina del conocimiento, actualizarse en las didácticas, las problemáticas sociales, profundizar en los marcos de análisis y narración, para desarrollar un modelo propio tanto de investigación, interpretación y difusión de la historia, en la formación de valores, como en el respeto, el reconocimiento del otro, la libertad, la justicia y muchos otros que contribuyan a la formación de la identidad y el carácter de un sujeto social.

Existen otros centros de reflexión sobre la didáctica de la historia, pero en su mayoría han trabajado la educación media y básica primaria (Carretero, 2007). Son pocos los estudios que han abordado la didáctica de la historia en la educación superior. Es el caso del profesor Vicente Benedito y de Joaquim Prats, quienes dirigen líneas de investigación de trabajos doctorales en la didáctica de la historia en las universidades españolas (Benedito, 1987; Prats, 2003). La Asociación Universitaria de Profesorado de Didáctica de las Ciencias Sociales fue creada por 
docentes universitarios españoles, quienes realizan cada año un simposio y tienen un órgano de difusión de los trabajos en didáctica tanto de la historia como de la geografía en España.

En el caso italiano se encuentran los trabajos de Ivo Mattozzi, quien se dedica ampliamente a investigar sobre la didáctica de la historia y la historiografía. El profesor Mattozzi, basado en el marco de referencia de la escuela italiana, plantea su preocupación por la ausencia de una conciencia de la génesis transpositiva del saber histórico, la cual incide en una deficiente enseñanza de la historia en la educación superior. La noción de transposición obliga a una nueva visión de la didáctica, pues considera el saber histórico y el texto que lo comunica como un problema y no como un supuesto previo (Mattozzi, 1999).

La escuela británica también ha desarrollado el estudio de la enseñanza y la didáctica de la historia en el nivel superior (Bourne, 1986). Uno de sus mayores exponentes en la actualidad es Alan Booth (2003), quien hizo una revisión en las últimas décadas del siglo XX sobre la enseñanza de la historia en la educación superior (Armstrong, 1999; Barnett, 1997; Booth, 2006) y ha encontrado que la historiografía y la narración histórica han sido abordadas de manera muy amplias, en contraste con las dimensiones pedagógicas, las cuales han estado ausentes del debate de lo público (Booth, 2006; Silver, 2006). Asimismo, esta escuela ha realizado estudios sobre el discurso político en la narración histórica y la enseñanza de la historia en la educación superior (Booth, 2004; Cannon, 1989). A este grupo le preocupan particularmente las políticas en educación superior frente a la separación de la figura docente-investigador, pues cree que si la didáctica de la historia no está alimentada desde la investigación, no puede aportar en la enseñanza de los estudiantes universitarios (Booth, 2009).

Por otro lado, en el campo anglosajón están los trabajos dirigidos por el profesor Sam Wineburg, quien lidera investigaciones en la didáctica de la historia y a su vez imparte clases de Enseñanza de la Historia en el Doctorado en Historia de la Educación de la Universidad de Stanford en Estados Unidos. Así mismo, ha escrito numerosos artículos y libros sobre la enseñanza de la historia en la educación superior (Wineburg, 2001a).

Por su parte, los educadores e historiadores en distintas latitudes fuera de la experiencia colombiana han hecho diversas propuestas para innovar la enseñanza de la historia (Pages, 2018). Algunas de ellas han introducido la metodología de investigación histórica en las aulas (Merchán, 2011), otras recurren al aprendizaje procedimental (Trepat, 1995) o por descubrimiento (Llonch, 2010) y otras a técnicas de la museografía, el periodismo y la literatura histórica (Parcero, 2010), la inicialización artística o científica (Hernández, 2010), y la difusión patrimonial (Martín, 2010). Carlos Barros (2007) sintetizó este proceso en un "nuevo paradigma educativo", centrado en las fuentes como el principal medio de estudio de la historia. De la misma forma, en países más cercanos a Colombia también se han desarrollado proyectos sobre la didáctica de la historia, como en la Universidad de Los Andes en Venezuela, que tiene una revista dedicada a los temas de la didáctica de las ciencias sociales. 
Otro gran polo de desarrollo de la enseñanza de la historia ha sido el grupo del profesor Mario Carretero, catedrático e investigador de la Flacso de Argentina. En este centro de estudio hacen investigaciones sobre temas del reconocimiento, como la identidad, la memoria y las representaciones vistas desde las narraciones históricas. En sus trabajos recientes, el grupo ha incorporado la enseñanza en el nivel superior, pues antes solo se centraba en la educación básica, media y primaria. Ahora se pregunta por los siguientes aspectos: ¿Cómo comprenden los alumnos los contenidos históricos y sociales, particularmente los que tienen que ver con la construcción de su identidad cultural o nacional?, ¿cómo se constituyen sus miradas sobre la significación moral del pasado?, ¿qué problemas y controversias plantea la enseñanza de la historia reciente en la escuela?, ¿cuál es la naturaleza de las representaciones sociales de los estudiantes?, ¿cuál es la relación de todo ello con la génesis de las ideologías y la comprensión infantil del mundo social? (Carretero, 2010).

En el caso colombiano, la didáctica de la historia ha sido analizada por el Grupo de Historia de las Prácticas Pedagógicas, que ha hecho varias publicaciones, tesis y monografías, en su mayoría sobre la trayectoria de las prácticas educativas y pedagógicas en la educación desde distintas disciplinas, no propiamente de la historia. Algunos informes y resultados de investigación son de utilidad en este estudio, sin que sean necesariamente el enfoque del trabajo, que se centra en la didáctica de la historia en la educación superior desde un estudio de caso, con una nueva forma de narración en la que se incluye el reconocimiento del otro.

En los años 70, los historiadores Jorge Orlando Melo y Jaime Jaramillo Uribe realizaron un informe desde las orientaciones teóricas y prácticas de la nueva historia y postulaban que los profesores de la enseñanza primaria y secundaria encargados de los cursos de Historia de Colombia debían tomar las orientaciones de la moderna historiografía. Otros trabajos temáticos en los que los historiadores incursionan en la enseñanza de la historia son los de Márquez (2009), quien menciona las debilidades teóricas de disciplinas como la historia:

\footnotetext{
Para entender e interpretar ese conflicto a la escuela le han faltado las características de una organización como un sistema abierto, dinámico, articulado, evolutivo, e histórico, y es aquí donde le cabe la responsabilidad de no haber asumido su papel protagónico en la búsqueda de salidas a la coyuntura. Las ciencias sociales, en especial la historia, la sociología, la antropología y la geografía en su condición epistemológica no han sido valoradas para tales propósitos. (Márquez, 2009, p. 46)
}

El Grupo de Enseñanza de la Historia de la Universidad Nacional, encabezado por José Gregorio Hernández, realizó una investigación que culminó en una publicación sugerente sobre la didáctica de la historia en las escuelas y colegios de Bogotá: Rutas pedagógicas de la historia en la educación básica de Bogotá (Rodríguez, 2005). En este proyecto, Rodríguez trabajó con el profesor Mauricio Archila, quien ha publicado también estudios de la enseñanza de la historia 
desde la perspectiva de la violencia y los discursos políticos (Archila, 1997). Sin embargo, no se ha realizado un estudio de la didáctica de la historia, sobre todo en la educación superior, aspecto que se aborda en este artículo.

Son pocos los trabajos que se han hecho sobre la educación superior, lo cual justifica que se profundice en su análisis. De las escasas investigaciones se destaca la que realizó el grupo de Javier Guerrero y Luis Weisner Gracia, de la Universidad Pedagógica Tecnológica de Colombia (UPTC), para la conmemoración del Bicentenario de la Independencia. Específicamente, realizaron una compilación de trabajos en torno a la siguiente pregunta: ¿para qué enseñar historia? (Guerrero, 2011), ensayos muy sugerentes que se incorporan a la reflexión de este trabajo de investigación. No obstante, muy poco se ha realizado en cuanto al desarrollo de las nuevas tecnologías en la enseñanza de la historia en la educación superior, por tal motivo este artículo hace una apuesta a este tipo de didácticas.

\section{Metodología de la propuesta didáctica}

\section{Ensayos como ejercicios narrativos didácticos en la reconstrucción de la historia}

Esta actividad se plantea en dos momentos: en el primero se establece como didáctica realizar un proceso de investigación a manera de monografía, conducente a presentar un artículo en una revista de divulgación académica universitaria indexada. En el segundo momento se intenta poner en marcha toda la propuesta de la "Historia Estelar" (Ojeda, 2018), incluso con un espacio académico propio denominado "Narraciones para el posconflicto".

En la primera actividad didáctica para la enseñanza de la historia en el nivel superior se quiso establecer un proceso de investigación con todos los parámetros de la metodología científica, en la que se solicitaba que los estudiantes tuvieran en cuenta los siguientes elementos:

1. Presentar el tema. Para ello los estudiantes deben tener en cuenta la redacción de mínimo dos párrafos en los que se establezca el tiempo y el espacio del objeto de investigación.

2. Ubicar el problema o la problemática del tema que van a investigar a partir del análisis de la bibliografía. Este análisis debe terminar en una muy buena pregunta que los estudiantes van a resolver. Para esta parte pueden utilizar entre cinco y nueve párrafos. Cabe aclarar que deben tener muy en cuenta el contexto y los antecedentes que identificaron desde las distintas investigaciones de tesis, artículos y libros.

3. Establecer el objetivo principal y el objetivo específico de acuerdo con la pregunta y al tema.

4. Señalar un estado del arte de todo lo que se ha escrito, indicando tendencias, enfoques, teorías y puntos de vista de los distintos autores e investigadores que 
han tratado estos temas o el tema de estudio escogido. Para esto deben utilizar entre 10 y 15 párrafos con las respectivas citas académicas.

5. Desarrollar el tema de acuerdo con la investigación propuesta, en la que resuelvan la pregunta y los objetivos. Todo esto debe estar muy bien argumentado y citado.

6. Tener una muy buena bibliografía.

A estos puntos se suman algunas aclaraciones que se dieron a los estudiantes: (1) tener en cuenta las normas APA para citar y escribir el artículo y (2) entregar un solo trabajo por el grupo, el cual debe tener en la portada los nombres de todos los integrantes'.

En esta propuesta didáctica se quería romper el esquema de hacer un examen final en el que se preguntaran de memoria tanto los hechos como los acontecimientos y sus relaciones contextuales, o que los estudiantes dieran cuenta de los procesos históricos con el formato memorístico. Se parte del hecho de que la memoria es importante, pero no para privilegiarla en un proceso valorativo, dado que en esta propuesta se privilegian las conexiones, el análisis, la interpretación, el poder de síntesis, las comparaciones, las inferencias, el pensamiento lógico y, sobre todo, la comprensión de un tema en particular. Los estudiantes realizan esta investigación y un video durante todo el semestre. En cada corte se van solicitando entregas previas de cada uno de los puntos y se corrigen, para lo cual se levanta un acta de entrega en la que se anotan todas las observaciones.

Al finalizar el semestre, los estudiantes y el docente siguen corrigiendo los mejores trabajos para pulir la redacción, el análisis y otros elementos, tanto de fondo como de forma, que es lo que se ha solicitado en las observaciones durante cada proceso evaluativo. Por ejemplo, en muchas de ellas el docente sugiere cambios, tanto en las preguntas como en el planteamiento del problema o en los objetivos, los cuales muchas veces los redacta junto con el estudiante. A su vez, en el proceso de consolidación de la información se hace un seguimiento y un trabajo en el que también participa el docente, de manera que se realiza un trabajo cooperativo, y luego se escogen, como premio, los mejores artículos, después de ser revisados exhaustivamente. Posteriormente, se prepara con mayor profundidad el análisis y las interpretaciones para consolidar un texto que se puede presentar a una revista indexada. Como producto final, en el tiempo intersemestral se realiza un trabajo extracurricular en el que se hacen otras adecuaciones para que el estudiante, junto con el profesor, queden como autores del escrito. En este punto es preciso aclarar que el 99 \% de los estudiantes nunca ha publicado en revistas indexadas y esto, para ellos, es la consolidación de su trabajo investigativo. Como solo se escoge uno cada semestre, los estudiantes tienen la posibilidad de presentar sus pro-

1 Estos parámetros fueron extraídos del aula virtual. Para esta clase, además de las clases presenciales, se dispuso un aula virtual en la plataforma de Moodle. Otro elemento que permite tener mejor interactividad con los estudiantes en la enseñanza de la historia. 
ductos a otras revistas por su cuenta, de hecho algunos han podido publicar sus resultados en revistas universitarias.

En la segunda propuesta didáctica se realizaron algunos cambios al desarrollo de la investigación. Estos consistieron en establecer como modelo la "Historia Estelar" y todo su desarrollo teórico concerniente tanto al tiempo como a la propuesta narrativa. De la misma manera que en la investigación, con el objeto de publicar un artículo en una revista universitaria, en esta propuesta de ensayo se menciona a los estudiantes cada una de las instrucciones desde el comienzo. En la primera clase del semestre se les presenta el syllabus, se les habla del seguimiento en el aula virtual mediante la plataforma Moodle y se da un tiempo para que los estudiantes escojan tanto el tema como el grupo con el que van a desarrollar la propuesta.

En esa primera clase y durante todo el semestre se hace énfasis en la psicología positiva, entendida como "el estudio científico de las experiencias positivas, los rasgos individuales positivos, las instituciones que facilitan su desarrollo y los programas que ayudan a mejorar la calidad de vida de los individuos, mientras previene o reduce la incidencia de la psicopatología" (Seligman 2005; Seligman \& Csikszentmihalyi, 2000). También es definida como el estudio científico de las fortalezas y virtudes humanas, las cuales permiten adoptar una perspectiva más abierta respecto al potencial humano, sus motivaciones y capacidades (Sheldon \& King, 2001), lo cual incluye virtudes cívicas e institucionales que guían a los individuos a tomar responsabilidades sobre su comunidad y promueve características para ser un mejor ciudadano (Contreras, 2006; Seligman \& Csikszentmihalyi, 2000).

En este espacio académico se cree que Colombia está permeada por la violencia y el conflicto, desde los medios de comunicación de todo tipo, desde las redes sociales, los espacios académicos y la cotidianidad. Todos los días se escuchan malas noticias y en muchos de los casos se privilegia esta percepción en las conversaciones. Por tal motivo, en esta propuesta del espacio académico "Narrativas para el posconflicto"2 se busca mostrar una faceta distinta de los diferentes acontecimientos que ocurren en el país. Se quiere contar la historia desde otra perspectiva y resaltar lo bueno, para lo cual se les dice a los estudiantes que hay que trabajar las lecturas con el ánimo de cambiar la mentalidad de nosotros mismos, para después impactar a los demás en el contexto del posconflicto.

Estas reflexiones han sido abordadas con mayor profundidad en el artículo que dio pie al concepto de la Historia Estelar Construcción de identidad desde las narraciones con una perspectiva psicohistórica (Ojeda, 2015). Con el fin de buscar la fórmula para acceder al cambio cultural o identitario desde las narraciones, se han hecho apuestas desde la psicología y su acercamiento a la identidad. Por ejemplo, en la propuesta se menciona desde la psicología:

2 Este espacio académico nació de la reflexión de la tesis doctoral Historia estelar: didáctica de las narrativas en la educación superior, en la cual se analizó una propuesta pedagógica y didáctica de la investigación, la cual se propuso en la Facultad de Ciencias Económicas y Sociales para ofertarla como electiva a todos los programas que tiene la Facultad. 
a partir de la investigación realizada en la línea "Didácticas pedagógicas" del Doctorado en Educación y Sociedad de la Universidad de La Salle, donde surge la pregunta ¿cómo se debe explicar y recuperar la memoria e identidad de una sociedad? Al establecer diálogos entre la historia, la filosofía y la psicología, hemos recurrido a un enfoque psicohistórico de tipo cualitativo, teórico-documental, donde se retoman conceptos que se pueden aplicar en una reflexión sobre la identidad, desde la narrativa para reconstruir la historia de un sujeto en relación con su entorno social. En esta medida, se encontró que la mejor manera de construir la memoria es teniendo en cuenta los hechos del pasado, la cotidianidad presente y el horizonte de expectativa del futuro; todo esto reunido en una trama narrativa que posibilita, desde la psicohistoria, una introspección para fomentar un cambio de mentalidad a nivel cultural. (Ojeda, 2015, p. 12)

La didáctica de la historia contempla la posibilidad de la acción en los lectores y su autorreflexión a partir de la interioridad de las narrativas propuestas con el modelo de la Historia Estelar. Para ello, los estudiantes tienen que efectuar las lecturas teóricas que sustentan esta propuesta, como también las metodológicas. Así, cuentan con los insumos para poder desarrollar la investigación y realizar toda la trama de la narración, en la que se busca reconstruir la historia y proponer nuevas formas, tanto de aprender como de enseñar a la sociedad sobre la historia del país, con un modelo didáctico que se ha explicado a lo largo del apartado.

En este modelo teórico de la Historia Estelar se cambia el concepto sincrónico de la temporalidad lineal por una temporalidad diacrónica, tal como funciona la red de conexiones del cerebro, en las que todas las dendritas y las neuronas permiten la interconectividad para producir conocimiento. Esta reflexión ha sido soportada desde los estudios de la neurociencia, en los cuales se ha podido identificar la construcción y percepción del tiempo histórico, del presente, el pasado y el futuro, elementos clave en la comprensión de la temporalidad para la construcción de la narrativa histórica.

\section{Marcos referenciales para la narración del pasado como mediación didáctica}

La percepción y el conocimiento de este primer acercamiento entre historia y narración sugieren determinar el concepto ricoeuriano de historia en un largo viaje por el relato histórico, el cual debe ayudar a comprender la identidad narrativa a partir de la estructura de la experiencia y las fuentes que se emplean en su construcción. De ahí se formulará la hipótesis de que la identidad narrativa, sea de una persona o de una comunidad, será el fin buscado de la nueva forma de hacer historia. En efecto, la construcción de la trama da un elemento más legible de las vidas humanas: cuando estas son interpretadas en función de las historias que la gente cuenta a propósito de ellos mismos, estas historias de vidas se hacen a su vez más inteligibles cuando se les aplican modelos narrativos, pequeñas tramas tomadas de las historias propiamente dichas (Ricoeur, 2006). 
La construcción de la trama posee un componente integrador de los agentes, fines y medios propios de la acción, en interacción con sus circunstancias, imprevistos y demás. El concepto admite todas las emociones que sentimos al efectuar la acción, como compasión, temor, padecimiento de efectos indeseados, entre otros componentes de la configuración, que Ricoeur (1999) ha caracterizado con el orden de concordancia sobre la discordancia. Al respecto asegura:

La narración pone de manifiesto en el orden sintagmático todos los componentes capaces de figurar en el cuadro paradigmático establecido por la semántica de la acción. Este paso de lo paradigmático a lo sintagmático constituye la transición misma de la mímesis I a la mímesis II como el fruto de la actividad configurativa. (Ricoeur, 1999, p. 132)

La narración en historia no puede ser un producto acabado, en contraposición a la fabricación, en la que la luz para juzgar el producto acabado la proporciona la imagen o modelo captado de antemano por el ojo del artesano. La luz que ilumina los procesos de la acción, y por tanto todos los procesos históricos, solo aparece en su final, frecuentemente cuando han muerto todos los participantes. La acción solo se revela plenamente al narrador, es decir, a la mirada del historiador, que siempre conoce mejor de lo que se trataba que los propios participantes. Aunque las historias son los resultados inevitables de la acción, no es el actor, sino el narrador, quien capta y hace la historia (Arendt, 1997, p. 215).

Es así como en la historia pueden existir múltiples relatos o narraciones de los acontecimientos; la historia no tiene fin y por lo tanto no puede producir resultados definitivos (Arendt, 1997, p. 30). Por esta razón se propone aquí otra forma de reconstruir los hechos históricos en la que no se tiene en cuenta una historia oficial, verdadera, sino una de tantas versiones o realidades de las acciones políticas de los sujetos que configuraron una sociedad en un tiempo determinado.

Tal cual lo observa Ricoeur en su análisis del modelo trágico de Aristóteles entre la dispersión episódica del relato y la unificación que se despliega al configurarlo, que es al mismo tiempo el acto poético (poiesis) mismo.

La diferencia esencial que distingue el modelo narrativo de cualquier otro modelo de conexión reside en el estatuto del acontecimiento, que en varias ocasiones hemos convertido en piedra de toque del análisis del sí, mientras que en un modelo de tipo causal, acontecimiento y ocurrencia permanecen indiscernibles. El acontecimiento narrativo es definido por su relación con la operación misma de la configuración, "participa de la estructura inestable de la concordancia-discordante, característica de la propia trama, es fuente de discordancia en cuanto surge, y fuente de concordancia en cuanto que hace avanzar la historia. (Ricoeur, 1999, p. 140) 
En la construcción de la trama, la contingencia tiene un papel inherente al mismo acontecimiento, es lo inesperado, lo sorprendente o lo probable, y esta solo se convierte en un integrante de la historia cuando es comprendida de forma indirecta en la totalidad temporal, donde los múltiples acontecimientos se convierten en uno solo. Esto también forma parte de la necesidad narrativa, cuyo efecto de sentido se presencia en la configuración en cuanto a tal. Entonces la necesidad narrativa convierte la contingencia física (identidad numérica, mismidad) en contingencia narrativa, en una simultaneidad constitutiva de ambas identidades.

En cuanto al reconocimiento, Ricoeur (2006) escribió en su última obra, desde una perspectiva fenomenológica, la reflexión filosófica de la alteridad. La exploración así emprendida concluye que la conciencia tiene como horizonte al otro y es necesariamente la reciprocidad para posibilitar en las acciones de la vida cotidiana el camino del reconocimiento de un nosotros (Ricoeur, 2006). Este tipo de marcos teóricos son los que acompañarán la reflexión conceptual en esta investigación.

\section{Ejercicio didáctico de la narración de la}

\section{historia a través de distintos formatos}

Con el fin de narrar la historia de una nueva forma se presentó como didáctica para los estudiantes de primer semestre de Negocios y Relaciones Internacionales de la Universidad de La Salle una actividad en la que ellos podían narrar la historia de un acontecimiento específico a partir de la creación de escritos narrativos de distintos formatos.

Para desarrollar esta actividad se les solicitó que hicieran una indagación previa de las temáticas que podían utilizar y las posibilidades de actuar dentro de la explicación de las distintas temáticas. Con esto se buscaba que los mismos estudiantes indagaran para crear un video documental y comprendieran el tema, pero también que al interpretar el papel y asumir el rol de algunos de los personajes históricos pudieran vivir en carne propia lo que sucedió en el pasado y así poder reconstruir la historia.

Este tipo de actividades didácticas responde a algunos modelos teóricos de enseñanza universitaria, como son la Teoría del Aprendizaje Significativo, propuesta por David Ausubel (1983). Él plantea dos modalidades de enseñanza: por recepción y por descubrimiento, que generan dos tipos de aprendizajes: memorísticos/repetitivos y significativos, respectivamente. De acuerdo con la teoría de Ausubel, para que se puedan lograr aprendizajes significativos es necesario que se cumplan tres condiciones:

1. Significatividad lógica del material. Significa que el material presentado tenga una estructura interna organizada, que sea susceptible de dar lugar a la construcción de significados. Es decir, importa no solo el contenido, sino la forma en que este es presentado.

2. Significatividad psicológica del material. Esto se refiere a la posibilidad de que el alumno conecte el conocimiento presentado con los conocimientos previos que ya 
ha incluido en su estructura cognitiva. El alumno debe contener ideas incluyentes en su estructura cognitiva, de lo contrario guardará la información en su memoria a corto plazo.

3. Actitud favorable del alumno. El aprendizaje no se puede dar si el alumno no quiere aprender. Este es un componente de disposiciones emocionales y actitudinales en el cual el docente solo puede influir a través de la motivación (Ausubel, 1983, p. 45).

Existen distintos espacios de aprendizaje y enseñanza de la historia, como por ejemplo los museos, los parques y las plazas, de carácter reales tangibles e intangibles, como los programas de multimedia y la realidad virtual. Pero en este trabajo se considera, como propone Bruner (1997), que los alumnos no son receptores pasivos del conocimiento, de tal manera que el aprendizaje se desarrolla por descubrimiento, usando recursos didácticos tangibles y adecuando el aula como un espacio donde se genera un ambiente motivador. En consecuencia, esta propuesta de aprendizaje considera la estructura y las condiciones cognitivas previas del aprendiz, así como el contexto sociocultural donde se desarrolla (Bruner, 1997). En este sentido, se tiene en cuenta las experiencias significativas de los estudiantes previas a desarrollar la actividad o la escogencia del tema.

\section{La mediación como eje del quehacer docente}

En este apartado se explicita la manera como se puede aplicar la propuesta didáctica de la narrativa en la reflexión de la disciplina histórica por medio del modelo de la Historia Estelar. Para ello es necesario tener en cuenta la mediación como último peldaño de la puesta en marcha del proyecto didáctico que se presenta.

El permanente progreso en el ejercicio de profesionalización docente implica, como lo afirma Korthagen (2001), pasar de un nivel de naturaleza intuitiva o inconsciente a niveles superiores de toma de consciencia, con la finalidad de profundizar en la comprensión de la propia función docente.

En este sentido, el docente está llamado a comprender su labor como un proceso consciente y planificado de mediación, entendida como una interacción en la que más allá de transmitir conocimientos, se ocupe de orientar los procesos y las estrategias de los estudiantes para alcanzar mejores aprendizajes, lo cual implica proponer retos que despierten la curiosidad y la atención, en un escenario de colaboración y buena disposición.

En efecto, como lo señala el profesor Tébar (2009), la labor de mediación exige despertar la curiosidad y la atención, de modo que al insinuar líneas de acceso al conocimiento y proponer los retos que posibiliten el desarrollo de los procesos de pensamiento, el docente podrá contribuir al aprendizaje autónomo del estudiante (Tébar, 2009). De hecho, muchos autores que han estudiado el funcionamiento del cerebro afirman que cuanto mayor es nuestra curiosidad por algo, mayor es la asimilación de los conocimientos compartidos (Teruel, 2013). 
Asimismo, hay que tener en cuenta que las capacidades inciden directamente en las competencias, los desempeños, pero sobre todo en los procesos de pensamiento. Los procesos cognitivos, tal como los define Tébar (2009), están constituidos por los siguientes factores básicos: el contenido sobre el que se opera, la operación mental implicada, el lenguaje con que se expresa la acción mental, la fase de procesamiento de la información y, por último, los niveles de complejidad, abstracción y eficacia de la operación.

Si se somete la estrategia didáctica de la Historia Estelar como modelo narrativo a los procesos mentales que agencia un mediador, se tienen que tener en cuenta las siguientes operaciones y estrategias para que docentes y estudiantes puedan ser conscientes de los procesos de formación que se desarrollan desde las actividades narrativas (tabla 1).

Tabla 1. Operaciones mentales y estrategias de activación

\begin{tabular}{ll}
\hline Operaciones mentales & Estrategias y técnicas de activación \\
\hline Identificación & Observar, subrayar, enumerar, contar, sumar, describir, preguntar, buscar. \\
\hline Comparación & Medir, superponer, transportar, contrastar. \\
\hline Análisis & $\begin{array}{l}\text { Identificar detalles, recuperar pros y contras, descubrir lo relevante, descomponer } \\
\text { en partes. }\end{array}$ \\
\hline Síntesis & Unir partes, seleccionar, abreviar, globalizar, ejemplificar mediante esquemas. \\
\hline Clasificación & $\begin{array}{l}\text { Elegir variables, seleccionar principios, criterios, parámetros, ordenar, jerarquizar, } \\
\text { usar cuadros, esquemas, diagramas, matrices. }\end{array}$ \\
\hline Codificación & Usar símbolos, signos, escalas, mapas. \\
\hline Decodificación & Asignar o crear significados, usar nuevas expresiones. \\
\hline Comprender & Identificar la esencia, expresar con otras palabras. \\
\hline Argumentar & Presentar razones para creer en algo. \\
\hline
\end{tabular}

Fuente: adaptado de Tébar (2009).

Desde este presupuesto, el maestro necesita saber cómo se produce el aprendizaje, qué mecanismos intervienen y, en consecuencia, seleccionar las mejores estrategias para promover su desarrollo y mejora continua. Es indispensable pensar el rol del docente como un científico que permanentemente indaga por mejores alternativas para hacer del conocimiento un aprendizaje vital que redunde en el desarrollo pleno de las capacidades de los estudiantes y contribuya a su formación integral. Por esto, para hablar de competencias se recurre a las reflexiones que hicieron las profesoras Carmen Amalia Camacho y Sandra Milena Díaz López (2013) en cuanto a estrategias didácticas, evaluación y competencias.

El historiador de hoy debe estimular, motivar, aventurarse e invitar a la aventura para que pueda ir más lejos y cruzar nuevos horizontes que le permitan enseñar no solo los proce- 
sos históricos, sino también las competencias para la vida en distintos escenarios. Es así que la historia, más allá de una disciplina, puede ser una mediación para obtener una mejor formación de la sociedad. En consecuencia, el historiador debe reflexionar su práctica pedagógica, de tal manera que posibilite un nuevo estilo de enseñanza basado en el análisis de los procesos cognitivos y de las competencias, y no solamente en los resultados de una historia colmada de datos y de memoria. De esta manera, el historiador como docente podría tener una visión más dinámica de la evaluación del potencial de aprendizaje y una confianza en los propios recursos para conseguir en los educandos mayores niveles de eficiencia (Tébar, 2009). Pensar que desde las narrativas hay una mediación para agenciar en los estudiantes formas de aprender distintas competencias para la vida y no solo el conocimiento por la erudición.

La Historia Estelar requiere de múltiples conexiones, que no se limitan a la competencia intelectual. Es una didáctica que permite formar en competencias sociales, empáticas, de valores, afectivas y sobre todo competencias mentales que pueden ser utilizadas en otros procesos de la vida.

Para efectos de este proyecto, la estrategia didáctica es entendida como un conjunto de procedimientos diseñados y estructurados en función de una meta de formación formulada a partir de los contenidos y las habilidades que un estudiante debe aprender en relación con un conocimiento específico. En este sentido, se asume que el saber está configurado por una serie de conocimientos universales que el sistema educativo selecciona para la formación de un ser humano particular en una sociedad y momento histórico determinado. De esta manera, el diseño de estrategias didácticas garantiza que el maestro se incorpore de manera activa a la gestión del currículo desde su práctica docente, en cuanto estas se planifican, desarrollan y evalúan a partir del alcance de un perfil de formación institucional previamente acordado y de unos objetivos específicos para cada momento de aprendizaje. Además, desde la óptica del estudiante (estrategia de aprendizaje), supone un compromiso de planeación, monitoreo y reflexión para la construcción de su propio saber y, principalmente, su capacidad de aplicarlo en su vida cotidiana (Camacho \& Díaz, 2013).

En relación con la enseñanza, el diseño de una estrategia supone reflexionar sobre la propia didáctica y, en función de este ejercicio, tomar intencionalmente decisiones oportunas sobre el planteamiento del proceso de mediación en el aula para lograr aprendizajes significativos. En esa medida, el docente está llamado a diseñar planes de acción que faciliten los procesos de aprendizaje y estén en consonancia con los propósitos de formación previstos.

Para rastrear en la acción la formación de competencias en el modelo narrativo de la Historia Estelar, se deben tener en cuenta los siguientes aspectos (Arendt, 1998): las acciones de enseñanza, las acciones de aprendizaje y las acciones cognitivas.

En cuanto a las acciones de enseñanza, se pueden distinguir todas aquellas que conducen a la comprensión de un tema específico que se quiere explicar. Por su parte, las acciones de aprendizaje hacen referencia a los métodos y didácticas que se utilizan para lograr dicho 
proceso. Y las acciones cognitivas son la interiorización, la comprensión y todas aquellas operaciones que exigen al cerebro las capacidades mentales.

Siguiendo el trabajo de Camacho y Díaz (2013), se toman prestados algunos diagramas de las acciones que se utilizaron en la puesta en marcha del modelo de la Historia Estelar (tabla 2).

Tabla 2. Modelo de estrategias didácticas

\begin{tabular}{|c|c|c|c|c|}
\hline Estrategia & Descripción & Utilidad & Pasos para desarrollarlo & $\begin{array}{c}\text { Acciones didácticas } \\
\text { asociadas }\end{array}$ \\
\hline $\begin{array}{l}\text { Aprendizaje } \\
\text { basado en } \\
\text { problemas }\end{array}$ & $\begin{array}{l}\text { Estrategia mediante } \\
\text { la cual un equipo } \\
\text { de estudiantes se } \\
\text { reúne para resolver } \\
\text { un problema } \\
\text { seleccionado } \\
\text { o construido } \\
\text { especialmente para } \\
\text { lograr determinados } \\
\text { objetivos de } \\
\text { aprendizaje. }\end{array}$ & $\begin{array}{l}\text { Permite la integración } \\
\text { del conocimiento } \\
\text { y posibilita su } \\
\text { transferencia y } \\
\text { aplicación. Permite } \\
\text { la creación de } \\
\text { nuevos escenarios } \\
\text { de aprendizaje y } \\
\text { promueve el trabajo } \\
\text { interdisciplinario. Su } \\
\text { diseño es flexible y } \\
\text { busca la participación } \\
\text { activa de estudiantes } \\
\text { y profesores. Favorece } \\
\text { la motivación del } \\
\text { estudiante, ya que ellos } \\
\text { adquieren un conjunto } \\
\text { de herramientas para } \\
\text { aplicar en diferentes } \\
\text { contextos de } \\
\text { desempeño. Permite } \\
\text { el desarrollo de } \\
\text { habilidades de relación } \\
\text { interpersonal y trabajo } \\
\text { en equipo. }\end{array}$ & $\begin{array}{l}\text { - Formular o establecer } \\
\text { - } \text { el problema. } \\
\text { - Recopilación de } \\
\text { la información } \\
\text { relacionada con el } \\
\text { problema. } \\
\text { - Identificar las } \\
\text { preguntas o incógnitas } \\
\text { asociadas al problema. } \\
\text { - Formulación de } \\
\text { respuestas o } \\
\text { resolución del } \\
\text { problema. } \\
\text { - Verificar la validez de } \\
\text { la respuesta o solución } \\
\text { propuesta. }\end{array}$ & $\begin{array}{l}\text { - Ideogramas. } \\
\text { - Lectura } \\
\text { autorregulada / } \\
\text { lectura dirigida. } \\
\text { - Producción } \\
\text { de textos } \\
\text { argumentativos. } \\
\text { - Ejercicios de } \\
\text { contrastación y } \\
\text { comparación. } \\
\text { - Análisis de } \\
\text { contexto. }\end{array}$ \\
\hline $\begin{array}{l}\text { Estudio de } \\
\text { caso }\end{array}$ & $\begin{array}{l}\text { Esta estrategia se } \\
\text { desarrolla relatando } \\
\text { una situación real, } \\
\text { en un contexto } \\
\text { semejante a aquel } \\
\text { en el que se pueden } \\
\text { desempeñar los } \\
\text { estudiantes y en el } \\
\text { que se debe tomar } \\
\text { decisiones. }\end{array}$ & $\begin{array}{l}\text { Permite al estudiante } \\
\text { desarrollar habilidades } \\
\text { de pensamiento } \\
\text { y contrastar sus } \\
\text { reflexiones con } \\
\text { las alternativas de } \\
\text { solución. Genera } \\
\text { disposición para el } \\
\text { trabajo cooperativo y la } \\
\text { capacidad de escuchar } \\
\text { y respetar propuestas } \\
\text { diferentes. Por último, } \\
\text { permite el desarrollo } \\
\text { de la creatividad y la } \\
\text { capacidad de tomar } \\
\text { decisiones. }\end{array}$ & $\begin{array}{l}\text { - Establecer el caso que } \\
\text { se va a estudiar. } \\
\text { - Recopilar toda } \\
\text { la información } \\
\text { relacionada con el } \\
\text { caso. } \\
\text { - Analizar la información } \\
\text { obtenida. } \\
\text { - Elaborar el informe } \\
\text { de acuerdo con } \\
\text { las características } \\
\text { específicas del caso y } \\
\text { las metas propuestas } \\
\text { al inicio del estudio. }\end{array}$ & $\begin{array}{l}\text { - Lectura } \\
\text { autorregulada. } \\
\text { - Producción de } \\
\text { textos. } \\
\text { - Descripción. } \\
\text { - Diseño, aplicación } \\
\text { y sistematización } \\
\text { - de encuestas. } \\
\text { - Ideogramas. }\end{array}$ \\
\hline
\end{tabular}




\begin{tabular}{|c|c|c|c|c|}
\hline Estrategia & Descripción & Utilidad & Pasos para desarrollarlo & $\begin{array}{c}\text { Acciones didácticas } \\
\text { asociadas }\end{array}$ \\
\hline $\begin{array}{l}\text { Aprendizaje } \\
\text { basado en } \\
\text { proyectos }\end{array}$ & $\begin{array}{l}\text { Pone a los } \\
\text { estudiantes en } \\
\text { situaciones que } \\
\text { los conduzcan } \\
\text { a recuperar, } \\
\text { comprender y } \\
\text { aplicar los diversos } \\
\text { aprendizajes como } \\
\text { un recurso para } \\
\text { proponer mejoras } \\
\text { en los distintos } \\
\text { contextos en los que } \\
\text { se desenvuelven. } \\
\text { Consiste en el } \\
\text { desarrollo de } \\
\text { experiencias } \\
\text { de aprendizaje } \\
\text { que involucran } \\
\text { al estudiante en } \\
\text { proyectos complejos } \\
\text { del mundo real. }\end{array}$ & $\begin{array}{l}\text { Favorece la creatividad } \\
\text { del alumno, su } \\
\text { capacidad de } \\
\text { trabajo autónomo, } \\
\text { su habilidad para } \\
\text { desarrollar proyectos } \\
\text { de investigación } \\
\text { y aplicación del } \\
\text { conocimiento } \\
\text { adquirido. Además le } \\
\text { permite desarrollar } \\
\text { su habilidad para el } \\
\text { trabajo productivo y } \\
\text { contextualizado. } \\
\text { Favorece las prácticas } \\
\text { innovadoras orientadas } \\
\text { al futuro y se centra } \\
\text { en actividades y } \\
\text { productos de utilidad } \\
\text { social o interés para los } \\
\text { organizadores. }\end{array}$ & $\begin{array}{l}\text { - Establecer el objetivo } \\
\text { del proyecto. } \\
\text { - Identificar acciones, } \\
\text { recursos y tiempos } \\
\text { necesarios para } \\
\text { realizar el proyecto. } \\
\text { - Asignar roles y } \\
\text { responsabilidades. } \\
\text { - Desarrollar el proyecto } \\
\text { - Evaluar el proyecto y } \\
\text { determinar su impacto. }\end{array}$ & $\begin{array}{l}\text { - Lectura } \\
\text { comprensiva. } \\
\text { - Análisis de } \\
\text { situaciones. } \\
\text { - Formulación de } \\
\text { propuestas. } \\
\text { - Diseño de } \\
\text { productos o } \\
\text { procesos. } \\
\text { - Evaluación de } \\
\text { productos y /o } \\
\text { procesos. }\end{array}$ \\
\hline
\end{tabular}

Fuente: adaptado de Camacho y Díaz (2013).

De todas estas estrategias didácticas, la que mejor se puede aplicar para enseñar las narrativas es el uso de proyectos finales de semestre como competencias investigativas. En consecuencia, a los estudiantes de Negocios y Relaciones Internacionales de primer semestre de la Universidad de La Salle se les pidió que, según el contexto, realizaran un estudio de un tema particular de las relaciones internacionales de Colombia con el mundo. Con esto se quería fomentar la indagación, la investigación y el conocimiento de la realidad internacional de su entorno, pues los estudiantes debían escoger un tema específico y realizar la recuperación de información para analizarla.

Todos estos proyectos se escogen desde el primer día de clase para que los estudiantes los vayan realizando durante todo el semestre. Al principio se les explica qué es un problema, cuál puede ser un estado del arte, cómo se determina una pregunta y, posteriormente, cómo se realizan los objetivos, tanto el general como los específicos. En este tipo de trabajos se privilegia el aprendizaje colaborativo, pues se hacen grupos de tres o cuatro personas con unas metas específicas y roles asignados. De esta manera, se favorece el cultivo de los valores sociales de mutua colaboración y responsabilidad compartida desde el concepto del entre nos.

Posteriormente, se les explica el marco conceptual y metodológico de la Historia Estelar para desarrollar una narrativa teniendo en cuenta los conceptos de trama, argumentación, implicación ideológica, tropos, metáforas y manejos de la temporalidad a la hora de emprender el camino de las narrativas y la redacción. Cuando los estudiantes han realizado toda la 
indagación, el análisis, la comparación y la síntesis, proceden a redactar un texto narrativo como proyecto final, en el cual deben aplicar todos los conceptos de la Historia Estelar.

Precisamente, en estos proyectos de escritura y producción de textos se agencian las distintas acciones de enseñanza. En primer lugar están los resúmenes o reseñas, los cuales permiten ubicar al estudiante dentro de la estructura o configuración general del saber que se va a aprender, al tiempo que posibilitan identificar la información importante, organizarla, integrarla y consolidarla (Camacho \& Díaz, 2013).

En segundo lugar, la producción de texto o ensayos potencian procesos de pensamiento como comprender, aplicar conceptos aprendidos, crear nuevos conocimientos sobre los temas establecidos y evaluar la validez de la información que se ha recogido, analizado y producido. Las habilidades de pensamiento que moviliza esta actividad pueden ser: codificar, resumir, integrar, argumentar, proponer y, por último, evaluar el proceso (Camacho \& Díaz, 2013). Asimismo, este tipo de proyectos de investigación en los que se crea una narrativa son ejercicios cognitivos que desarrollan competencias en recursos gramaticales, escriturales, inferenciales, argumentativos e ideológicos. Por último, estos proyectos permiten desarrollar capacidades para sintetizar información, construir y socializar conocimiento, evaluar información presentada en un texto, argumentar posiciones propias, trabajar de forma independiente y autorregulada (Camacho \& Díaz, 2013).

Los estudiantes cargan todos los trabajos a la plataforma Moodle que tiene la universidad para que sean evaluados siguiendo los parámetros de las acciones cognitivas, para lo cual se utiliza como instrumento de seguimiento los informes periódicos según las etapas de investigación. Por ejemplo, durante el semestre se realizan tres cortes de evaluación, los cuales exigen un reporte de seguimiento y unas notas. Para ello, en cada uno de los cortes se va exigiendo a los estudiantes que entreguen adelantos tanto de la investigación como del seguimiento de la escritura y la narración.

Según Carmen Amalia Camacho y Sandra Milena Díaz (2013), la evaluación de un proceso de formación debe ser asumida como un ejercicio multidimensional que involucra diversos momentos, perspectivas e instrumentos. En este sentido, se debe superar la mirada tradicional que la asocia con la medición y la calificación, de tal forma que se constituya en un proceso más humanizado y situado en función del contexto y las necesidades de quienes participan en ella (Camacho \& Díaz, 2013).

En consecuencia, la evaluación no se puede concebir como la fase terminal del proceso, es decir, como un promedio de resultados (evaluación sumativa). Por el contrario, debe estar integrada a él, de tal modo que permita identificar las necesidades iniciales del estudiante y, en concordancia con ellas, oriente el diseño del plan de formación o acompañamiento, es decir, que permita establecer un diagnóstico inicial (evaluación diagnóstica). Además, debe 
acompañar todo el proceso, de modo que permita una reflexión constante y un mejoramiento continuo, tanto de los aprendizajes como de las prácticas de enseñanza. Esto implica que la retroalimentación ofrecida por el docente debe ser pertinente y efectiva para apoyar a los estudiantes en la regulación de sus aprendizajes y para generar planes que conlleven el diseño de nuevas acciones para cualificar la práctica (Camacho \& Díaz, 2013).

Tabla 3. Rúbrica de evaluación de las narrativas en la Historia Estelar

\begin{tabular}{|c|c|c|c|c|c|c|}
\hline \multirow{2}{*}{$\begin{array}{l}\text { Criterios de } \\
\text { evaluación }\end{array}$} & \multirow[b]{2}{*}{ Indicadores de evaluación } & \multicolumn{5}{|c|}{ Escala de valoración } \\
\hline & & 1 & 2 & 3 & 4 & 5 \\
\hline \multirow{5}{*}{$\begin{array}{l}\text { Análisis crítico } \\
\text { (procedimental) }\end{array}$} & $\begin{array}{l}\text { 1. Identifica y analiza de manera crítica los diferentes } \\
\text { argumentos propuestos en los textos presentados para su } \\
\text { lectura. }\end{array}$ & & & & & \\
\hline & 2. Identifica una problemática con sus diferentes variables. & & & & & \\
\hline & $\begin{array}{l}\text { 3. Establece una pregunta seguido de los objetivos que dan } \\
\text { cuenta de las variables identificadas. }\end{array}$ & & & & & \\
\hline & 4. Desarrolla un marco de referencia y un contexto. & & & & & \\
\hline & 5. Explicita el marco metodológico de la investigación. & & & & & \\
\hline \multirow{5}{*}{$\begin{array}{l}\text { Claridad } \\
\text { conceptual } \\
\text { (conceptual) }\end{array}$} & $\begin{array}{l}\text { 1. Demuestra claridad conceptual al momento de socializar } \\
\text { sus argumentos en relación con las lecturas asignadas en la } \\
\text { Historia Estelar. }\end{array}$ & & & & & \\
\hline & 2. Identifica y aplica el concepto de trama narrativa. & & & & & \\
\hline & 3. Identifica y aplica el concepto de argumentación. & & & & & \\
\hline & 4. Identifica y aplica el concepto de las ideologías. & & & & & \\
\hline & 5. Identifica y aplica las metáforas y los tropos. & & & & & \\
\hline \multirow{5}{*}{$\begin{array}{l}\text { Cumplimiento } \\
\text { con los } \\
\text { compromisos } \\
\text { (actitudinal) }\end{array}$} & $\begin{array}{l}\text { 1. Cumple puntualmente con los compromisos establecidos: } \\
\text { asistencia a clase. }\end{array}$ & & & & & \\
\hline & $\begin{array}{l}\text { 2. Entrega de productos acordados en cada etapa de la } \\
\text { escritura. }\end{array}$ & & & & & \\
\hline & $\begin{array}{l}\text { 3. Realiza los ajustes de sus procesos y productos de } \\
\text { acuerdo con la retroalimentación realizada. }\end{array}$ & & & & & \\
\hline & 4. Sustenta el trabajo de manera escrita y expositiva. & & & & & \\
\hline & $\begin{array}{l}\text { 5. Socializa la narrativa tanto en clase como en otros } \\
\text { escenarios. }\end{array}$ & & & & & \\
\hline
\end{tabular}

Continúa tabla.. 


\begin{tabular}{|c|c|c|c|c|c|c|}
\hline \multirow{2}{*}{$\begin{array}{l}\text { Criterios de } \\
\text { evaluación }\end{array}$} & \multirow[b]{2}{*}{ Indicadores de evaluación } & \multicolumn{5}{|c|}{ Escala de valoración } \\
\hline & & 1 & 2 & 3 & 4 & 5 \\
\hline \multirow{5}{*}{$\begin{array}{l}\text { Comportamiento } \\
\text { ético } \\
\text { (actitudinal) }\end{array}$} & $\begin{array}{l}1 \text { Evidencia un comportamiento respetuoso, receptivo, } \\
\text { proactivo y solidario en el desarrollo de las sesiones de clase. }\end{array}$ & & & & & \\
\hline & $\begin{array}{l}\text { 2. Favorece el desarrollo de todas las dimensiones del } \\
\text { ser humano mediante la interacción, el respeto y el } \\
\text { reconocimiento de símismo y de un nosotros. }\end{array}$ & & & & & \\
\hline & $\begin{array}{l}\text { 3. Desarrolla los valores sociales de mutua colaboración y } \\
\text { responsabilidad compartida. }\end{array}$ & & & & & \\
\hline & $\begin{array}{l}\text { 4. Agencia la responsabilidad individual y la responsabilidad } \\
\text { de grupo. }\end{array}$ & & & & & \\
\hline & $\begin{array}{l}\text { 5. Permite validar las ideas individuales y el consenso } \\
\text { colectivo. }\end{array}$ & & & & & \\
\hline
\end{tabular}

Observaciones:

Fuente: elaboración propia.

\section{Conclusiones}

La educación se debe comprender como una red de posibilidades, una configuración de particularidades, tanto teóricas como pedagógicas, también didácticas, cognitivas y de otro tipo de enfoques o posturas, que pueden participar en el proceso de formación de la sociedad y que deben funcionar como un todo ordenado. Para lograr esto, como en la narración histórica, es necesario tener una trama bien diseñada y un argumento o propósito que guíe el procedimiento y la acción.

En esta propuesta que parte de la didáctica de la Historia Estelar no deben importar los contenidos más que los valores. Se deben tener en cuenta las subjetividades, como señala Alfonso Torres (2006):

En la subjetividad confluyen dimensiones culturales y psíquicas reconocidas desde diferentes disciplinas sociales y campos de estudio como la antropología, el psicoanálisis, la psicología social, la historia y los estudios culturales. Además, destaca los imaginarios culturales, las representaciones sociales, la memoria social, las creencias, las ideologías, el inconsciente, el pensamiento, los conocimientos, los valores, las emociones y los sentimientos, las voluntades y las visiones de futuro. (p. 65)

Todos estos elementos funcionan como estrellas polares que posibilitarán la conexión estelar y la configuración de otra forma de educación estelar. En este marco de educación, 
la formación mediada por lo estelar debe promover la integridad, la justicia, la pluralidad, la diversidad, el respeto por las diferencias, la inclusión, el perdón, un nosotros, conceptos que, más que palabras, se puedan llevar a la acción. Así como en la Historia Estelar se busca una interpretación y un análisis de los hechos para comprenderlos y apropiarlos en la cotidianidad, la Educación Estelar debe ser una constelación de sentido y comprensión de la realidad, vista desde distintas perspectivas, en las que se pueden construir y reconfigurar constelaciones de sentido para la vida práctica.

La Educación Estelar debe ser, entonces, un proceso de desarrollo y formación sociocultural en la que se potencien valores para generar una mejor sociedad. Se debe establecer un modelo cultural en el que primen las experiencias de resiliencia, superación, mediación, motivación y liberación, pero, sobre todo, una sanidad emocional y espiritual para poder transformar el entorno e impactar a otras generaciones con expectativas de futuro.

En la Educación Estelar debe primar lo humano, el sentido de autorrealización a través de la comprensión de los otros, y en la construcción de un nosotros. Formar en los estudiantes deseos de vivir, soñar, sentir, pensar, analizar y comprender. Una educación en la que se enseña responsabilidad, capacidad para vivir en comunidad, asumiendo responsabilidades para ser mejores personas y no únicamente para acumular y tener objetos y cosas materiales. Debe primar la confianza en las personas, en sus procesos individuales, y el conocimiento de sí mismas, para que se puedan relacionar con los demás en una pluralidad de un nosotros diverso.

En la Didáctica Estelar se deben tener en cuenta las conexiones de todo tipo, las relaciones y las redes, pues estas ayudan a configurar un orden social. Se debe privilegiar el estudio del cerebro, de sus posibilidades de conexión neuronal en la construcción del conocimiento, pero, sobre todo, en la formación de unas mejores personas, con pensamientos positivos, adaptativos a los estilos cognitivos, reflexivos y comprensivos.

El aprendizaje, mediado por el conocimiento del cerebro, permite que la sociedad sea más consciente de sus propios ritmos, a saber, la intervención de cada uno de sus órganos en los sentidos, las emociones, los afectos y, sobre todo, las relaciones. En este tipo de Didáctica Estelar es mejor organizar configuraciones de sentido para la formación —como si fueran constelaciones - de aprendizaje significativo, que simplemente cumplir con unos planes de estudios, unas metas de contenidos sin ninguna relación con la realidad del entorno en que viven los estudiantes. Por eso se debe enseñar a conocer la mente, su cerebro, conocerse a sí mismo para poder tener la capacidad de empatía con los demás.

En la Educación Estelar todos son copartícipes de la formación de los demás, pues se privilegian las redes, el trabajo colaborativo, la cooperación y el que los estudiantes no esperen a que el docente sea el depositario del conocimiento. La Educación Estelar privilegia la 
reflexión y el empoderamiento del estudiante, la autonomía, el éxito y las buenas prácticas, pues implica señalar lo positivo en los demás y revisar cómo se puede potenciar mutuamente los estudiantes, precisamente, para provocar en los demás una motivación en este camino de formación, de autocontrol, de paciencia, de autorreflexión, de diversión y de pasión por el conocimiento.

En este sentido, la Educación Estelar debe ser diversa en todo sentido, desde el plano teórico, epistémico, pedagógico y, sobre todo, didáctico. Se deben combinar distintas metodologías y estrategias de aprendizaje, salir de la rutina, traer al aula la tecnología, las nuevas formas de conocimientos, romper con los esquemas. Asimismo, implica acercarse a los estudiantes en sus dificultades, en sus formas particulares de aprender, reconocer sus debilidades y sus problemas de aprendizaje. También es necesario reconocer la capacidad que tienen para ser creativos y adaptar las estrategias de enseñanza, para formar un clima agradable de aprendizaje, en donde se reconocen los errores y los problemas, al tiempo que se es creativo e innovador para salir adelante con respeto y cuidado de los demás.

En definitiva, la Educación Estelar acoge todo tipo de pedagogía cognitiva y neurocientífica, en la que se promueven la creatividad, las capacidades artísticas, las emociones, los sentidos, el despertar de la sensibilidad por el conocimiento, por la espiritualidad en el crecimiento del ser humano en su máxima expresión psicológica, donde se forme con valores, con amor, respeto y mucho cuidado por la vida y los seres vivos.

\section{Agradecimientos}

El autor agradece a la Universidad de La Salle por su apoyo en la realización de este artículo.

\section{Declaración de divulgación}

El autor declara que no existe ningún potencial conflicto de interés relacionado con el artículo.

\section{Financiamiento}

El autor no declara fuente de financiamiento para la realización de este artículo.

\section{Sobre el autor}

Robert Ojeda-Pérez es Ph.D. en educación y sociedad de la Universidad de La Salle, magíster en Historia de la Universidad de Los Andes, especialista en Pedagogía y Didáctica, e historiador de la Pontificia Universidad Javeriana. Es miembro de número de la Academia de Historia de Bogotá.

https://orcid.org/0000-0002-1227-7854-Contacto: rojeda@unisalle.edu.co 


\section{Referencias}

Arendt, H. (1997). ¿Qué es la política? Paidós Ibérica.

Armstrong, M. (1999). Historical and contextual perspectives on benchmarking in higher education. En H. Smith, M. Armstrong \& S. Brown (eds.), Benchmarking and threshold standards in higher education. Kogan Page.

Ausubel, D. (1983). Teoría del aprendizaje significativo. CEIF.

Ávila Ruiz, R. M., Rivero Gracia, M. P., \& Domínguez Sanz, P. L. (Coord.). (2010). Metodología de investigación en didáctica de las ciencias sociales. AUPDCS.

Barnett, R. (1997). Higher education: a critical business. Open University Press.

Barros, C. (2007). Propuestas para el nuevo paradigma educativo de la historia. http://www.h-debate. com/cbarros/spanish/publicacionesdigitales/varios/egonpe.pdf

Benedito, V. (1987). Introducción a la didáctica. Fundamentación teórica y diseño curricular. Ed. Barcanova.

Booth, A. (2004). Rethinking the scholarly: developing the scholarship of teaching and learning in history. Arts \& Humanities in Higher Education, 3, 247-266.

Booth, A. (2006). Perspectives on the research-teaching relationship in history. http://www.hca.heacademy.ac.uk/assets/hca/documents/case_Studies/snas/booth.doc

Booth, A. (2009). Pedagogy and the practice of academic history in late-twentieth century Britain. Rethinking History, 13(3), 317-344.

Bourne, J. (1986). History at the universities. History, 71, 54-60.

Bruner, J. (1997). La educación, puerta de la cultura. Visor

Cannon, J. (1989). Teaching history at university. The History Teacher, 22, 245-75.

Carretero, M. (2007). Documentos de identidad. La construcción de la memoria histórica en un mundo global. Paidós.

Carretero, M., \& Castorina, J. A. (2010). La construcción del conocimiento histórico: enseñanza, narración e identidades. Paidós.

Guerrero García, C. A. (2011). La incidencia de las reformas educativas en la enseñanza de la historia en Colombia, 1973-2007 [tesis de maestría, Universidad Nacional de Colombia]. Repositorio Institucional. http://bdigital.unal.edu.co/6257/

Hernández Carretero, A. M., \& Daniel Abel, S. (2010). Los análisis paleopalinológicos como recurso didáctico para la consecución de aprendizajes integradores. En R. M. Ávila Ruiz, M. P. Rivero Gracia, \& P. L. Domínguez Sanz (Coord.), Metodología de investigación en didáctica de las ciencias sociales (pp. 651655). AUPDCS.

Korthagen, F. A. (2001). Linking practice and theory. The pedagogy of realistic teacher education. LEA.

Llonch Molina, N. (2010). El método por descubrimiento en la enseñanza de ciencias sociales: ejemplificación y análisis. En R. M. Ávila Ruiz, M. P. Rivero Gracia, \& P. L. Domínguez Sanz (Coord.), Metodología de investigación en didáctica de las ciencias sociales (pp. 597-606). AUPDCS.

Márquez Quintero, M. (2009). Enseñanza de la historia del conflicto armado en Colombia: fundamentos para la construcción de propuestas para su enseñanza en el ámbito universitario. Revista Latinoamericana de Estudios Educativos, 5(2),205-230. https://www.redalyc.org/articulo.oa?id=1341/134116861009

Martín Cáceres, M., Cuenca López, J. M., \& Estepa Giménez, J. (2010). La didáctica del patrimonio. Metodología y resultados de investigaciones en el ámbito educativo formal, no formal e informal. En R. M. Ávila Ruiz, M. P. Rivero Gracia, \& P. L. Domínguez Sanz (Coord.), Metodología de investigación en didáctica de las ciencias sociales (pp. 641-645). AUPDCS. 
Mattozzi, I. (1999). La transposición del texto historiográfico: un problema crucial de la didáctica de la historia. Teoría y Didáctica de las Ciencias Sociales, 4, 27-56.

Melo, J. O. (1999). Medio siglo de historia colombiana: notas para un relato inicial. Revista de Estudios Sociales, 4, 9-22. https://doi.org/10.7440/res4.1999.01

Merchán, F. J. (2011). El control de la conducta del alumnado en el aula: ¿un problema para la práctica de la investigación escolar? Revista de Investigación en la Escuela, 73, 53-63.

Ojeda Pérez, R. (2015). Claves para la comprensión de una historia estelar: interpretación, comprensión, hermenéutica y diversidad política. Praxis PedaGógica, 15(17), 67-82. doi:http://dx.doi.org/10.26620/ uniminuto.praxis.15.17.2015.67-82

Ojeda Pérez, R. (2018). De la Historia antigua a la Historia Estelar, un viaje a través del tiempo. Revista Cambios y Permanencias, 9(2), 527-551.

Ojeda Pérez, R., \& Lizcano Roa, J. (2015). Construcción de identidad desde las narraciones con una perspectiva psicohistórica. Revista Aletheia, 7(2). http://aletheia.cinde.org.co/index.php/ALETHEIA/article/view/252

Pagès Blanch, J., \& Santisteban Fernández, A. (2018). La enseñanza de la historia. Historia y Memoria, 17, 11-16. https://dx.doi.org/10.19053/20275137.n17.2018.8283

Parcero Torre, C. (2010). Cómo mejorar el interés por la historia indagando las posibilidades que proporciona el género biográfico. En R. M. Ávila Ruiz, M. P. Rivero Gracia, \& P. L. Domínguez Sanz (Coord.), Metodología de investigación en didáctica de las ciencias sociales (pp. 567-575). AUPDCS.

Posada López, R. P. (2010). La enseñanza de la historia: perspectivas y retos en la educación superior. Estudio de caso: enseñanza de la historia en la Licenciatura en Ciencias Sociales de la Universidad de Caldas. Revista Latinoamericana de Estudios Educativos, 6(2), 179-203. https:/www.redalyc.org/ articulo.oa?id=1341/134126048009

Prats, J. (2003). Líneas de investigación en didáctica de las ciencias sociales. História \& Ensino. http://www. ub.edu/histodidactica/images/documentos/pdf/lineas_investigacion_didactica_ciencias_sociales.pdf

Sánchez Quintanar, A. (1995). Enseñar historia en la universidad y fuera de ella. Perfiles Educativos, 68. https://www.redalyc.org/articulo.oa?id=132/13206809

Seligman, M. \& Csikszentmihalyi, M. (2000). Positive Psychology: an introduction. American Psychologist, 55(1), 5-14.

Ricoeur, P. (1999). La lectura del tiempo pasado: memoria y olvido. Edición Universidad Autónoma de Madrid.

Ricoeur, P. (2006). Caminos de reconocimiento. Fondo de Cultura Económica.

Rodríguez, J. G. (ed.). (2005). Rutas pedagógicas de la historia en la educación básica de Bogotá. Universidad Nacional de Colombia; Instituto de Investigación Educativa y Desarrollo Pedagógico.

Tébar, L. (2009). El profesor mediador del aprendizaje. Magisterio.

Torres Carrillo, A. (2006). Subjetividad y sujeto: perspectivas para abordar lo social y lo educativo. Revista Colombiana de Educación, 50, 86-103. https://doi.org/10.17227/01203916.7741

Trepat, C.-A. (1995). Procedimientos en Historia. Un punto de vista didáctico. Graó.

Tuning, A. (2007). Reflexiones y perspectivas de la educación superior en América Latina. http://tuningacademy.org/wp-content/uploads/2014/02/TuningLAIII_Final-Report_SP.pdf

Tuning, A. (2013). Educación superior en América Latina: reflexiones y perspectivas en historia.

Wineburg, S. (2001). Knowing, teaching, and learning history: national and international perspectives. American Historical Association. 